\title{
Carbon nanotube synthesis from propane decomposition on a pre-treated Ni overlayer
}

\author{
J SENGUPTA, S K PANDA and C JACOB* \\ Materials Science Centre, Indian Institute of Technology, Kharagpur 721 302, India
}

MS received 2 July 2008

\begin{abstract}
Growth of carbon nanotubes (CNTs) was performed by atmospheric pressure chemical vapour deposition (APCVD) of propane on Si(111) with a pre-treated Ni overlayer acting as a catalyst. Prior to the growth of CNTs, a thin film of $\mathrm{Ni}$ was deposited on $\mathrm{Si}(111)$ substrate by evaporation and heat treated at $900^{\circ} \mathrm{C}$. The growth of nanotubes was carried out at $850^{\circ} \mathrm{C}$ using propane as a source of carbon. Distribution of the catalyst particles over the Si substrate was analysed before and after heat treatment by atomic force microscopy (AFM). The X-ray diffraction (XRD) pattern of the grown material revealed that they are graphitic in nature. Field emission scanning electron microscopy (FESEM) was used to investigate the growth process and it was found that a catalytic particle was always situated at the tip of the tube thus implying a tip growth mechanism. Evidence for the presence of radial breathing mode from multi-wall nanotubes (MWNTs) in the grown sample was obtained from micro-Raman analysis. Finally, high-resolution transmission electron microscopic (HRTEM) analysis confirmed that the graphene layers of the CNTs are well ordered with typical 0.34 nm spacing.
\end{abstract}

Keywords. Carbon nanotubes; Ni-catalysed growth; APCVD; AFM; HRTEM; micro Raman spectroscopy.

\section{Introduction}

An unique combination of electronic, thermal, mechanical and chemical properties (Dai et al 1996; Saito et al 1997; Tans et al 1998; Chen et al 1999; Qian et al 2000) have made CNTs a centre of attraction in the field of nanoscale research since their discovery in 1991 by Iijima (Iijima 1991). CNT-based field-emission devices exhibit outstanding emission characteristics (Heer et al 1995; Kim et al 2000) and remarkable current stability (Dean and Chamala 1999; Zhu et al 1999) as an outcome of the high aspect ratio, mechanical stiffness and electrical conductivity of the nanotubes (Wong et al 1997; Yao et al 2000). Due to the large surface area, low resistivity and chemical stability of nanotubes along with the capability to grow directly onto desired substrates, CNTs emerge as a potential electrode material for electrochemical energy storage devices and supercapacitors (Leroux et al 1999; Nutzenadel et al 1999; Frackowiak et al 2000).

Although many basic experiments are centred on SWNTs but such materials produce at high temperatures and are hard to manipulate into useful configurations. Conversely, MWNTs grow at lower temperatures directly onto substrates in useful geometries (Huang et al 1998; Ren et al 1998). Compared to SWNTs, the larger wall thicknesses of MWNTs lead to larger diameters, higher

*Author for correspondence (cxj14_holiday@yahoo.com) stiffness, and highly improved electrical conductivity; which make MWNTs a suitable candidate for coldcathode electron field emission, nanoporous membranes, low-friction nanobearings, ultra-sensitive electrometers and other applications (Shiflett and Foley 1999; Cummings and Zettl 2000; Roschier et al 2001).

The synthesis of CNTs can be grouped into the following categories (Iijima 1993; Thess et al 1996; Journet et al 1997; Meyyappan et al 2003): arc discharge, chemical vapour deposition, plasma method, laser ablation, etc. Among the above methods, CVD method is simple and easy to implement, and has been widely used because of its potential advantage to produce a large amount of CNT growing directly on a desired substrate with high purity, large yield and controlled alignment, whereas the nanotubes must be collected separately in the other growth techniques (Iijima 1993; Thess et al 1996; Journet et al 1997; Kong et al 1998; Meyyappan et al 2003).

To achieve a controllable growth of the CNTs, an understanding of their growth mechanism is of importance. The general growth process of CNTs by CVD is based on the following mechanism proposed by Baker et al (1972) known as the vapour-liquid-solid (VLS) mechanism. In this mechanism, liquid catalytic particles at high temperature absorb carbon atoms from the vapour to form a metal-carbon solid state solution. When this solution becomes supersaturated, carbon precipitates at the surface of the particle in its stable form and lead to the formation of a carbon tube structure. Depending on the position of 
the catalyst particle, base and tip growth mechanisms have been proposed (Tesner et al 1972; Oberlin et al 1976; Tibbets 1984).

The metal clusters acting as a catalyst for CNT growth by CVD can be produced by different methods, e.g. by vapour or sputter deposition of a thin metallic layer on the substrate, by saturation of porous materials by metals, by deposition of solutions of substances containing catalyzing metals or by introduction of organometallic substances into the reactor (Jeong et al 2002; Tu et al 2002; Huang et al 2003; Bertoni et al 2004). Prior to CNT synthesis, high temperature hydrogen treatment is an important step in order to produce contamination free catalyst particles and for the removal of the oxides that may exist over catalyst surface (Takagi et al 2007).

In this paper, we have discussed the formation of carbon nanotubes using APCVD over a pre-heated Ni catalyst on $\mathrm{Si}(111)$ substrate using propane as a source of carbon. AFM study was performed on the Ni catalyst, before and after heat treatment to observe the change in morphology due to heating. FESEM and HRTEM were used to characterize the growth morphology and structure of the nanotubes. Compositional information was obtained by XRD and micro-Raman spectroscopy.

\section{Experimental}

Atmospheric pressure chemical vapour deposition of CNTs was carried out by catalytic decomposition of propane on $\mathrm{Si}(111)$ wafers with a pre-treated $\mathrm{Ni}$ overlayer in a hotwall horizontal CVD reactor using a resistance-heated furnace (ELECTROHEAT EN345T).

The Si (111) substrates were ultrasonically cleaned with acetone and deionized water prior to Ni film deposition. For Ni deposition, the sample was loaded in a vacuum system (Hind Hivac: Model 12A4D) and pumped down to a base pressure of $10^{-5}$ Torr and Ni thin films (thickness $\sim 20 \mathrm{~nm}$ ) were deposited by evaporation of metallic nickel (purity, 99.994\%).

The substrates were then loaded into a quartz tube furnace, pumped down to $10^{-2}$ Torr and backfilled with flowing argon. When the furnace temperature stabilized at $900^{\circ} \mathrm{C}$, the samples were annealed in hydrogen atmosphere for $10 \mathrm{~min}$. Finally, the reactor temperature was brought down to $850^{\circ} \mathrm{C}$ and the hydrogen was turned off, thereafter propane was introduced into the gas stream at a flow rate of $200 \mathrm{SCCM}$, for $1 \mathrm{~h}$ for CNT synthesis.

A nanonics Multiview $1000^{\mathrm{TM}}$ system in AFM mode was used to image the surface morphology of the Ni layer before and after heat treatment with a quartz optical fibre tip in tapping mode. Samples were also characterized by a Rigaku X-ray diffractometer (ULTIMA III) with $\mathrm{CuK} \alpha$ source and $\theta-2 \theta$ geometry to analyse the crystallinity and phases of grown species. Micro Raman measurements were carried out at room temperature in a backscattering geo- metry using a $488 \mathrm{~nm}$ air-cooled $\mathrm{Ar}^{+}$laser as an excitation source for compositional analysis. FESEM (ZEISS SUPRA 40) and HRTEM (JEOL JEM 2100) were employed for examination of the morphology and microstructure of the CNTs.

\section{Results and discussion}

\subsection{AFM analysis}

Figure 1 shows the 3-D AFM image of the distribution of the as deposited catalyst particle over the Si (111) substrate. The AFM image reveals that the initial film consists of Ni clusters instead of a continuous layer. As $\mathrm{Ni}$ atoms interact more strongly with each other than they do with the Si substrate hence the catalyst deposition proceeds via island nucleation and coalescence, in accor-

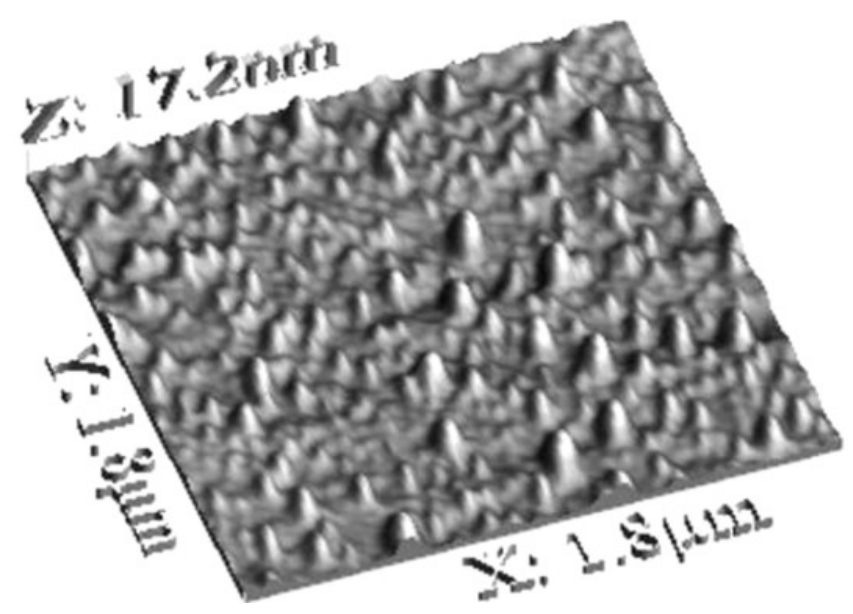

Figure 1. A 3D AFM image of the as-deposited Ni film, showing the clusters forming at the early stages of growth.

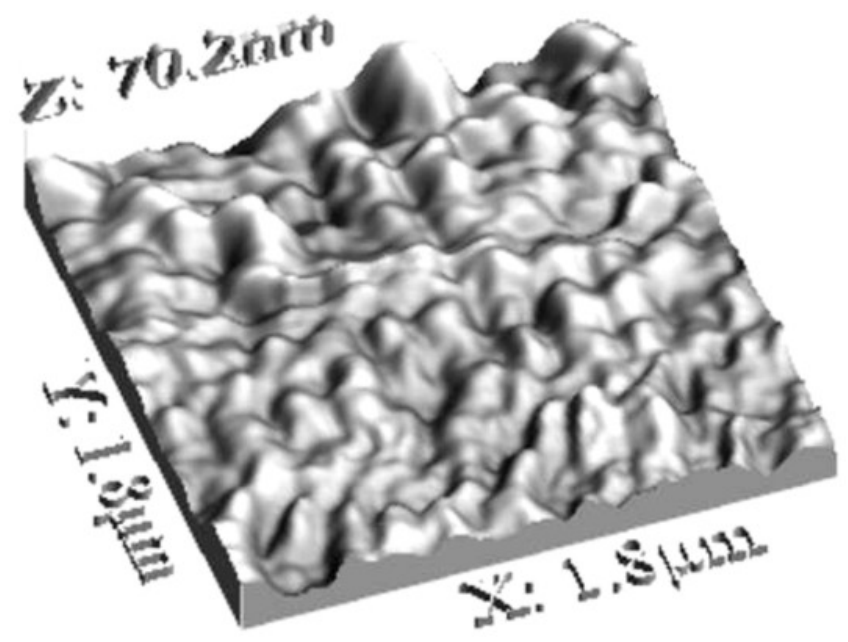

Figure 2. A 3D AFM image of $\mathrm{Ni}$ islands formed upon annealing the substrate at $900^{\circ} \mathrm{C}$. 
dance with the Volmer-Weber mode (Tiller 1991). After deposition of $\mathrm{Ni}$, the sample was annealed in hydrogen for $10 \mathrm{~min}$ at $900^{\circ} \mathrm{C}$. This heat treatment results in the formation of $\mathrm{Ni}$ islands as confirmed by AFM image in figure 2. Heating above a certain temperature causes clusters to coalesce and form macroscopic islands. This process is based on cluster diffusion and depends on their density and diffusion constant, at a given substrate temperature. Cluster diffusion terminates when the island shape is of minimum energy for the specific annealing conditions (Jak et al 2000). These clusters act as a catalyst surface for nanotube growth.

\subsection{X-ray diffraction analysis}

A XRD measurement was carried out using $\mathrm{Cu} \mathrm{K} \alpha$ radiation $(\lambda=1.54059 \AA)$ to examine the structure of the CNTs and the resulting $\theta-2 \theta$ scan is shown in figure 3 . The peak at $26.3^{\circ}$ is the characteristic graphitic peak arising due to the presence of multiwall carbon nanotubes (MWNTs) in the sample with inter-planar spacing of $0.34 \mathrm{~nm}$ (Cao et al 2001). The interlayer spacing of MWNT $(d(002)=0.344 \mathrm{~nm})$ in the radial direction is similar to that of graphite $(d(002)=0.335 \mathrm{~nm})$. The slightly larger $d$ spacing compared to that of graphite is related to the stacking disorder in these nanostructures. The peak near $43.6^{\circ}$ is attributed to the (101) plane of the nanotube (Chatterjee et al 2003). The peaks at $28.5^{\circ}$ and $58.9^{\circ}$, however, did not originate from the MWNTs and are attributed to (111) and (222) planes, respectively of the $\mathrm{Si}$ substrate.

\subsection{FESEM analysis}

Field emission scanning electron microscopy (FESEM) was employed for the analysis of the morphology and

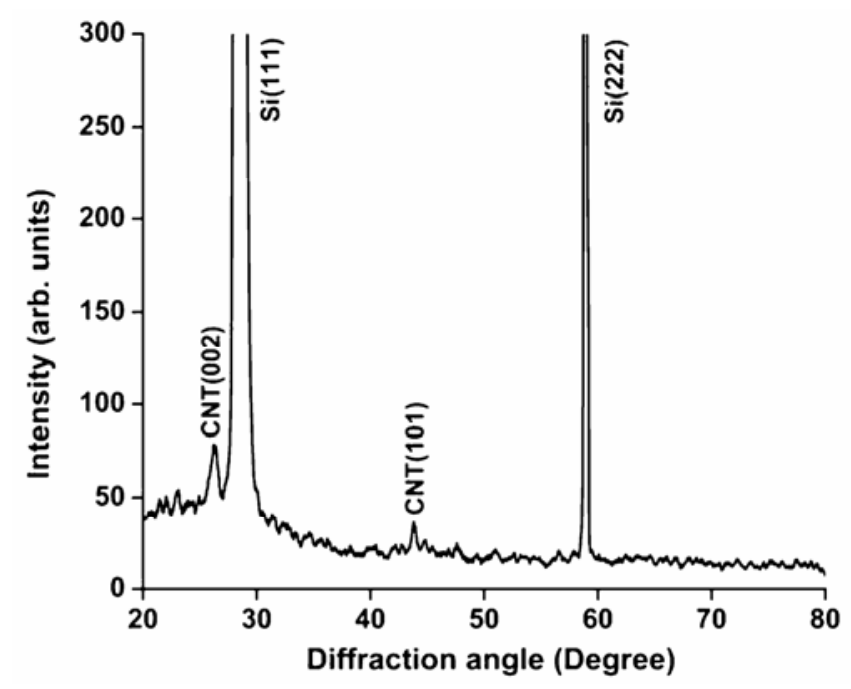

Figure 3. X-ray diffraction spectra of MWNTs grown on $\mathrm{Si}$ (111) substrate. density of MWNTs. Figures 4a and $\mathrm{b}$ are the FESEM micrographs showing the surface morphology of Ni-coated $\mathrm{Si}$ wafers after propane exposure. High-aspect ratio nanostructures are observed on the Ni film surfaces; there is no observable growth of these structures on bare Si substrates. The area density of the deposited MWNTs was high and the MWNT structures had randomly oriented spaghetti-like morphology. We note that in figure 4a the number of small tubes is much higher than the big ones and the tube diameter is in the range of $15-100 \mathrm{~nm}$. Figure $4 \mathrm{~b}$ shows the high magnification image of the small tubes with a narrow diameter distribution around $20 \mathrm{~nm}$. In many cases, small bright catalyst particles were detected at the tip of the tubes. This suggests that the tip growth mechanism is likely to be responsible for the MWNT synthesis under the present conditions.

\subsection{HRTEM analysis}

High-resolution transmission electron microscopy (HRTEM) was used to characterize the growth morphology and structure of the nanotubes. Figure 5a shows highresolution transmission electron microscopic (HRTEM) images of a MWNT grown from the Ni nanoparticle. It can be clearly seen that the CNT is well graphitized with an inner diameter of about $14-17 \mathrm{~nm}$ and outer diameter, $38-42 \mathrm{~nm}$. The thickness of the tube wall lies in the range of $12-14 \mathrm{~nm}$, which suggests that the tube wall is composed of $\sim 30-40$ graphitic layers. In the same figure, various defects and bamboo-like structures (diaphragms) inside the CNT can also be observed. The distance between two neighbouring graphitic walls is about $0.34 \mathrm{~nm}$ (inset); in agreement with the interplanar distance of CNT $(d 002)$ of $0.344 \mathrm{~nm}$ as obtained from XRD. Figure $5 \mathrm{~b}$ shows the selected area diffraction (SAD) pattern from the MWNT shown in figure 5a. There are diffuse halos due to the amorphous carbon film on the copper grid and sharp rings due to the MWNT. The diffraction rings corresponding to a layer distance of 0.34 and $0.17 \mathrm{~nm}$, are considered to be of the graphite (002) and (004) diffractions, respectively. The diffraction rings corresponding to layer distances of 0.20 and $0.12 \mathrm{~nm}$ can also be seen in figure $5 \mathrm{~b}$. The former corresponds to (101) and the latter to (110) of graphite.

\subsection{Raman analysis}

Micro-Raman spectroscopy provides more details of the quality and structure of the materials produced. The Raman spectra were taken in the backscattering geometry on the sample as grown. Figure 6 shows the room temperature Raman spectra of the MWNT material at a laser excitation wavelength of $488 \mathrm{~nm}$. The spectrum is divided into two main zones: the low frequency region from 150 $800 \mathrm{~cm}^{-1}$ (figure 6a) and the high frequency zone from 

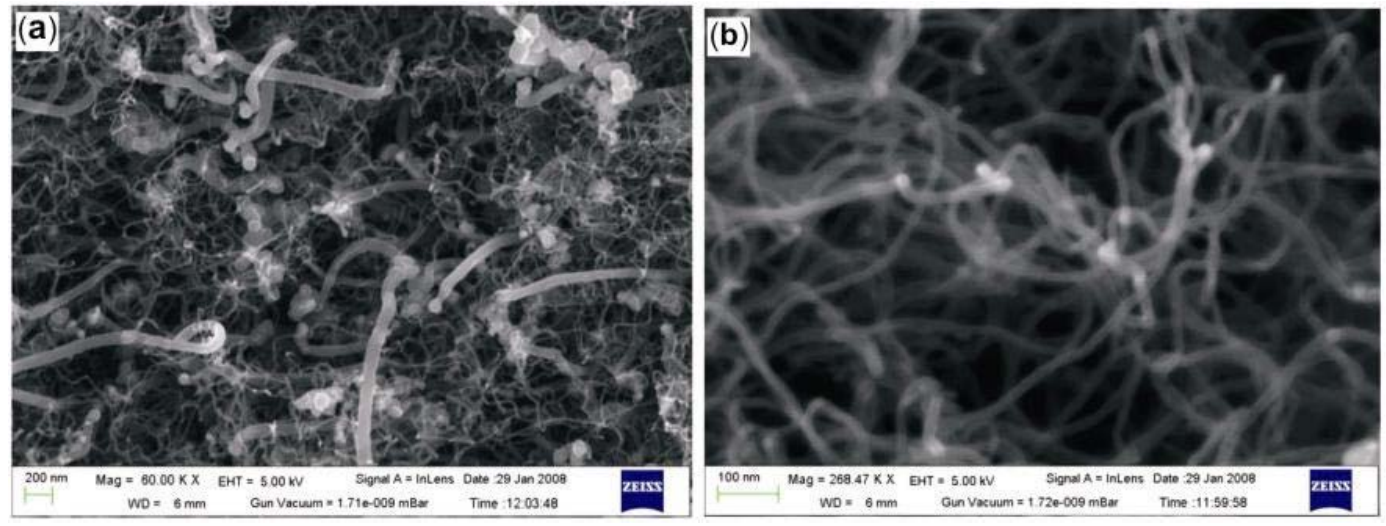

Figure 4. a. FESEM micrograph of the as-grown MWNTs deposited using the APCVD method and b. high magnification FESEM image of the small tubes.
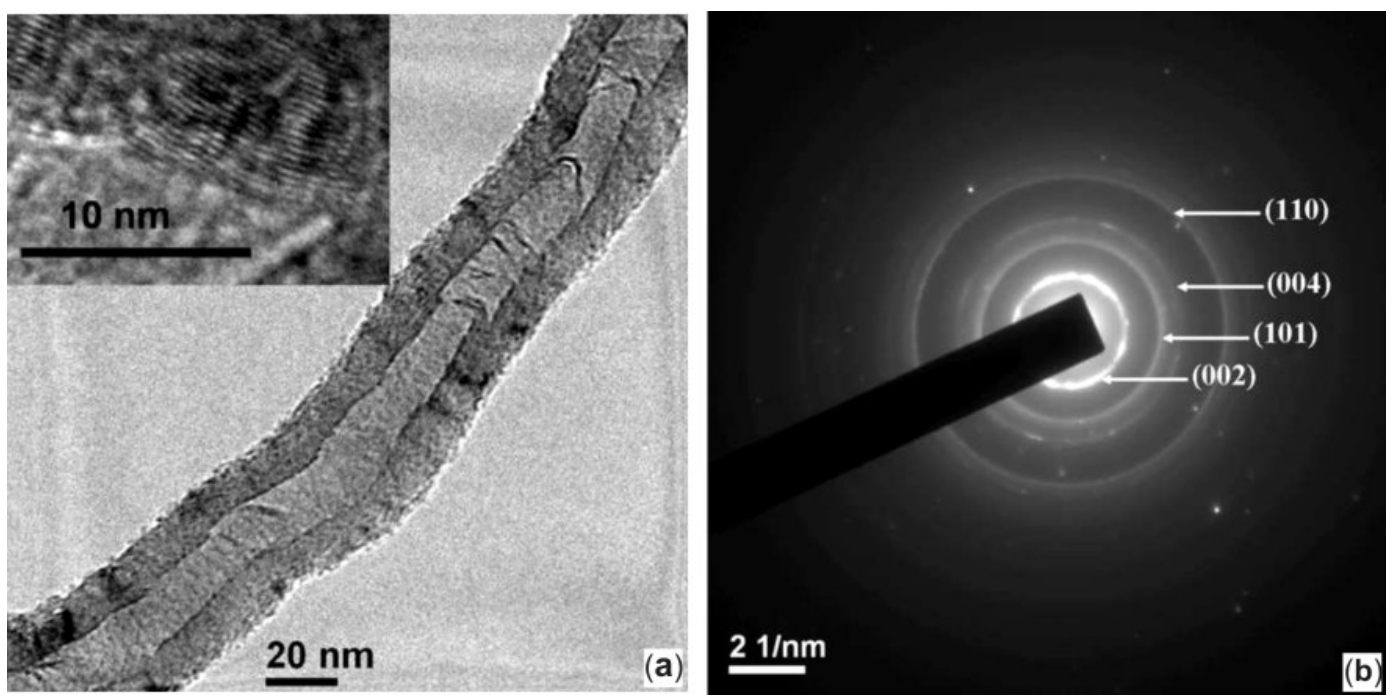

Figure 5. a. HRTEM image of the well-graphitized MWNT grown from the Ni using the APCVD method. (Inset) The fringe spacing between two carbon layers of MWNTs is about $0.34 \mathrm{~nm}$ and $\mathbf{b}$. the selected area diffraction (SAD) pattern from the MWNT shown in figure 5a.

$1200-1700 \mathrm{~cm}^{-1}$ (figure $6 \mathrm{~b}$ ). The vibrations of CNT originate from the curvature induced strain due to misalignment of the $\pi$-orbitals of adjacent coupled carbon atoms. These vibrations are reflected in the Raman peaks.

Evidence for the presence of radial breathing mode vibrations in this sample is obtained from the low wave number range of the spectrum (figure 6a). The peaks located at $220 \mathrm{~cm}^{-1}$ and $286 \mathrm{~cm}^{-1}$ are due to the radial breathing mode of MWNT (Jinno et al 2004). This mode has $A_{1 \mathrm{~g}}$ symmetry and all the carbon atoms move in phase in the radial direction creating breathing like vibration of the entire tube (Zhao et al 2002). The frequency of RBM is directly linked to the innermost tube diameter by the relation

$$
\omega_{\mathrm{RBM}}=223 \cdot 75 / d,
$$

where $d$ is the innermost tube diameter and $\omega_{\mathrm{RBM}}$ the wave number in units of $\mathrm{nm}$ and $\mathrm{cm}^{-1}$ (Jinno et al 2004).
The peaks at $220 \mathrm{~cm}^{-1}$ and $286 \mathrm{~cm}^{-1}$ originate due to the radial breathing mode of MWNT bundle with inner-core diameter of $1 \mathrm{~nm}$ and $0.78 \mathrm{~nm}$, respectively.

The two main peaks observed in the high frequency zone (figure 6b) are the so-called $D$ - and $G$-lines (Eklund et al 1995). The $G$-line appears at $1567 \mathrm{~cm}^{-1}$ and corresponds to the $E_{2 \mathrm{~g}}$ mode i.e. the stretching mode of the $\mathrm{C}-\mathrm{C}$ bond in the graphite plane and demonstrates the presence of crystalline graphitic carbon.

The $D$-line, at $1350 \mathrm{~cm}^{-1}$, originates from disorder in the $\mathrm{s} p^{2}$-hybridized carbon and can be due to the presence of lattice defects in the graphite sheet that makes up carbon nanotubes. The position of the $D$ band for MWNT can be expressed as $\omega_{\mathrm{D}}=1285+26 \cdot 5 E_{\text {laser }}$ (Wei et al 2003). In our case, $E_{\text {laser }}=2.54 \mathrm{eV}$ resulting in $\omega_{\mathrm{D}}=1352.3 \mathrm{~cm}^{-1}$, which is in agreement with our observed peak position and the FWHM is $43 \mathrm{~cm}^{-1}$, whereas Dillon et al (2004) have stated the full width at half-maximum (FWHM) of 
the $D$-band of amorphous carbon and nano-crystalline graphite at $488 \mathrm{~nm}$ excitation as $\sim 57$ and $86 \mathrm{~cm}^{-1}$. On the basis of these two characteristics (frequency and linewidth), we can conclude that the presence of the $D$ band in the sample is due to the MWNT. However, a recent Raman analysis of multiwall nanotubes (MWNT) suggests that the $D$ band is an intrinsic feature of the Raman spectrum of MWNTs, and they are not necessarily an indication of a disordered wall structure (Rao et al 2000).

The degree of graphitization is an indicator of the carbon nanotubes disorder level, and is evaluated by the intensity ratio of the $D$ to $G$ peaks $\left(R=I_{\mathrm{D}} / I_{\mathrm{G}}\right)$. The intensity ratio derived from figure $6 \mathrm{~b}$ is $R=I_{\mathrm{D}} / I_{\mathrm{G}}=0 \cdot 2$, indicating that the grown MWNT is highly crystalline in nature.
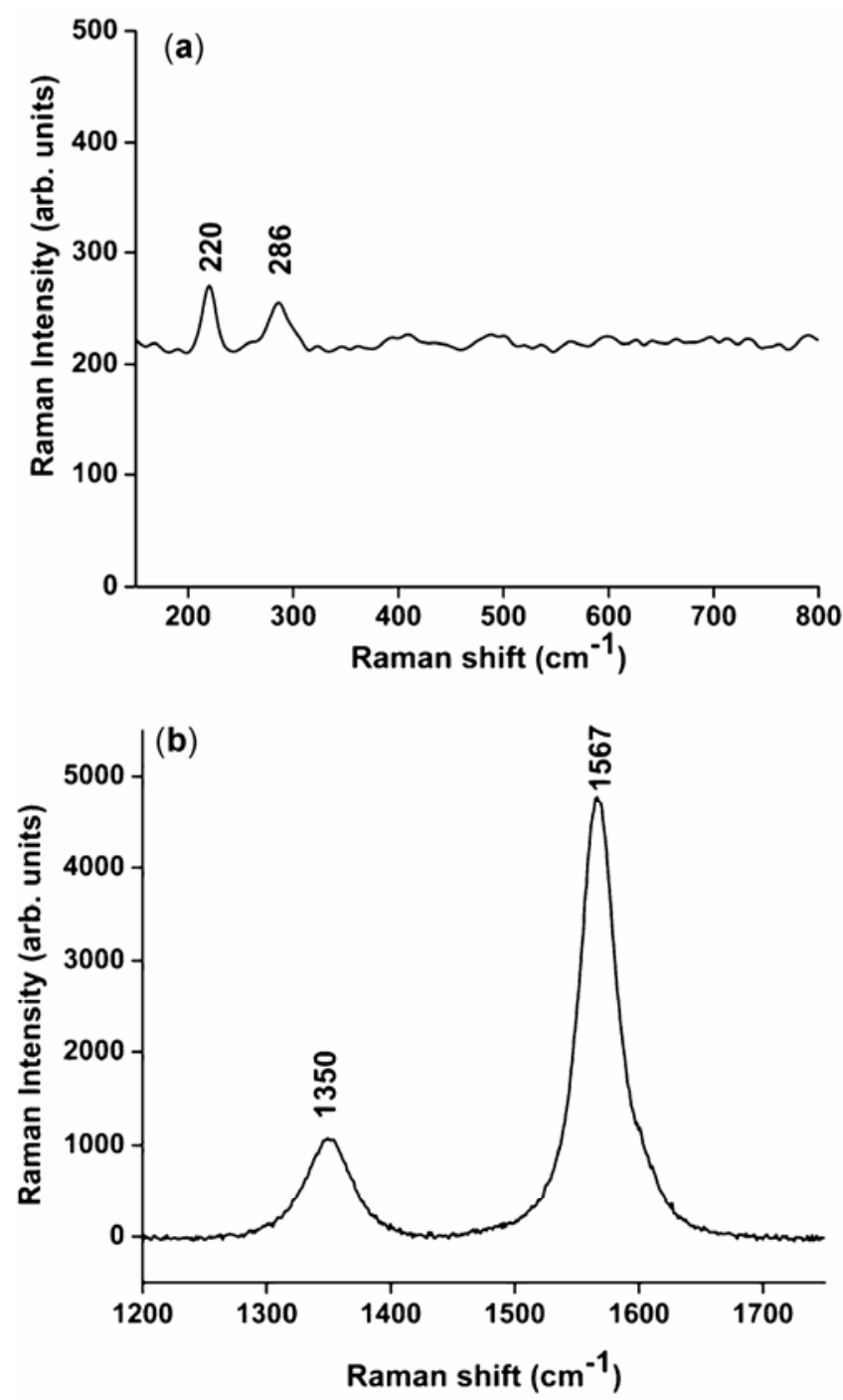

Figure 6. a. Low frequency Raman spectra (488 nm excitation) of a MWNT film grown by APCVD on Si showing the presence of RBM and $\mathbf{b}$. high frequency Raman spectra (488 nm excitation) of a MWNT film grown by APCVD on $\mathrm{Si}$.

\section{Conclusions}

We have grown MWNT by catalytic decomposition of propane on $\mathrm{Si}$ with a pre-treated $\mathrm{Ni}$ overlayer at $850^{\circ} \mathrm{C}$ by atmospheric chemical vapour deposition. At the growth temperature of $850^{\circ} \mathrm{C}$, the catalyst particles coalesce on the substrate as confirmed by AFM studies. The XRD results suggest that MWNTs with good graphitization have been grown. Micro Raman analysis shows that radial breathing modes are present in the spectrum, generated from small diameter MWNTs. The FESEM images lead to the evidence that, in this experiment, MWNTs growth with $\mathrm{Ni}$ catalyst was primarily by the tip-growth mechanism. Lastly, HRTEM studies prove that the grown materials are carbon nanotubes and give a layer spacing of $0.34 \mathrm{~nm}$. Since the catalyst particles are produced and deposited on a substrate using a dry process, the current method is applicable to any types of substrates. Moreover, many types of nanoparticles can be produced by simple evaporation. This will help in the optimization of catalyst materials for future studies.

\section{Acknowledgement}

We are grateful to Dr A Roy, Department of Physics, Indian Institute of Technology Kharagpur, for her help in the Raman measurement.

\section{References}

Baker R T K, Barber M A, Harris P S, Feates F S and Waite R J 1972 J. Catal. 2651

Bertoni G, Cepek C, Romanato F, Casari C S, Bassi A L, Bottani C E and Sancrotti M 2004 Carbon 42423

Cao A, Xu C, Liang J, Wu D and Wei B 2001 Chem. Phys. Lett. 34413

Chatterjee A K, Sharon M, Banerjee R and Spallart M N 2003 Electrochim. Acta 483439

Chen P, Wu X, Lin J and Tan K L 1999 Science 91285

Cummings J and Zettl A 2000 Science 289602

Dai H J, Hafner J H, Rinzler A G, Colbert D T and Smalley R E 1996 Nature 384147

Dean K A and Chamala B R 1999 Appl. Phys. Lett. 753017

Dillon A C, Yudasaka M and Dresselhaus M S 2004 J. Nanosci. Nanotech. 4691

Eklund P C, Holden J M and Jishi R A 1995 Carbon 33959

Frackowiak E, Metenier K, Bertagna V and Beguin F 2000 Appl. Phys. Lett. 772421

Heer W A de, Chatelain A and Ugarte D 1995 Science 2701179

Huang Z P, Xu J W, Ren Z F, Wang J H, Siegal M P and Provencio P N 1998 Appl. Phys. Lett. 733845

Huang Z P et al 2003 Appl. Phys. Lett. 82460

Iijima S 1991 Nature $\mathbf{3 5 4} 56$

Iijima S 1993 Mater. Sci. Eng. B19 172

Jak M J J, Konstapel C, Kreuningen A van, Verhoeven J and Frenken J W M 2000 Surf. Sci. $\mathbf{4 5 7} 295$

Jeong H J et al 2002 Chem. Phys. Lett. 361189 
Jinno M, Bandow S and Ando Y 2004 Chem. Phys. Lett. 398 256

Journet C et al 1997 Nature 388756

Kim J M, Choi W B, Lee N S and Jung J E 2000 Diamond Relat. Mater. 91184

Kong J, Cassell A M and Dai H J 1998 Chem. Phys. Lett. 292 567

Leroux F, Metenier K, Gautier S, Franckowiak E, Bonnamy S and Beguin F 1999 J. Power Sources 81317

Meyyappan M, Delzeit L, Cassell A and Hash D 2003 Plasma Sources Sci. Technol. 12205

Nutzenadel C, Zuttel A, Chartouni D and Schlapbach L 1999 Electrochem. Solid-State Lett. 230

Oberlin A, Endo M and Koyama T 1976 J. Cryst. Growth 32 335

Qian D, Dickey E C, Andrews R and Rantell T 2000 Appl. Phys. Lett. 762868

Rao A M, Jorio A, Pimenta M A, Dantas M S S, Saito R, Dresselhaus G and Dresselhaus M S 2000 Phys. Rev. Lett. 84 1820

Ren Z F, Huang Z P, Xu J W, Wang J H, Bush P, Siegal M P and Provencio P N 1998 Science 2821105

Roschier L, Tarkiainen R, Ahlskog M, Paalanen M and Hakonen P 2001 Appl. Phys. Lett. 783295
Saito Y et al 1997 Nature 389554

Shiflett M B and Foley H C 1999 Science 2851902

Takagi D, Hibino H, Suzuki S, Kobayashi Y and Homma Y 2007 Nano Letts 72272

Tans S J, Verschueren A R M and Dekker C 1998 Nature 393 49

Tesner P A, Robinovich E Y, Rafalkes I S and Arefieva E F 1972 Carbon 8435

Thess A et al 1996 Science 273483

Tibbets G G 1984 J. Cryst. Growth 66632

Tiller W A 1991 The science of crystallization: Microscopic interfacial phenomena (Cambridge, UK: Cambridge University Press) p. 172

Tu Y, Huang Z P, Wang D Z, Wen J G and Ren Z F 2002 Appl. Phys. Lett. 804018

Wei J, Jiang B, Zhang X, Zhu H and Wu D 2003 Chem. Phys. Lett. 376753

Wong E W, Sheehan P E and Lieber C M 1997 Science 277 1971

Yao Z, Kane C L and Dekker C 2000 Phys. Rev. Lett. 842941

Zhao X, Ando Y, Qin L, Kataura H, Maniwa Y and Saito R 2002 Phys. B323 265

Zhu W, Bower C, Zhou O, Kochanski G and Jin S 1999 Appl. Phys. Lett. 75873 